\title{
Una Nueva Propuesta Didáctica para la Enseñanza Universitaria de Química Orgánica
}

\author{
M.M. Blanco, M.E. Hedrera, M.R. Dal Maso y L.R. Orelli \\ Univ. de Buenos Aires, Fac. de Farmacia y Bioquímica, Depto. de Química Orgánica, Junín No956, \\ (1113) Buenos Aires - Argentina (e-mail: Iorelli@ffyb.uba.ar)
}

\begin{abstract}
Resumen
En este trabajo se discute la reformulación de las actividades en clases de problemas y de trabajos prácticos de laboratorio de química orgánica básica y de los materiales impresos utilizados en las mismas. El objetivo de esta reformulación es despertar el interés y la participación de los alumnos e introducirlos al trabajo científico y profesional. Se presenta como ejemplo una sección de la Guía de Problemas y de Actividades de Laboratorio correspondiente a la unidad Síntesis Orgánica. Se realizaron observaciones de ambos tipos de clases, en las que se trabajó con los recursos didácticos tradicionales y con los nuevos. El análisis de las observaciones indicó que la mayoría de los alumnos se involucraron activamente en la nueva propuesta y que aumentó la motivación de los estudiantes, estimuló la consulta y análisis de textos y favoreció la aplicación de los contenidos conceptuales a situaciones problemáticas concretas.
\end{abstract}

Palabras claves: enseñanza universitaria, estrategias de enseñanza, resolución de problemas, química orgánica

\section{A New Didactic Proposal for University Teaching of Organic Chemistry}

\begin{abstract}
The study discusses the reformulation of problem activities in classes and laboratory work in basic organic chemistry courses and of the printed materials use in these courses. The objective of these reformulations was to awaken student interest and participation in scientific and professional activities. As an example, a section of the Guide to Problems and Laboratory Activities corresponding to the unit Organic Synthesis is presented. Observations are made on both types of classes, in which work was done with traditional didactic methods. Analysis of the observations suggested that the majority of students became actively involved with the new proposal. It is concluded that the proposed activities improved the motivation of the students, stimulated consultation and study texts, and favored the application of the conceptual contents to real problems situations.
\end{abstract}

Keywords: university teaching, teaching strategies, problem solving, organic chemistry

Nota: este artículo está tomado de "Información Tecnológica" [ISSN 0716-8756], vol. 13(2), 165-168 (2002) INTRODUCCIÓN 
Las materias Química Orgánica I, II y III son parte del Ciclo Común de las carreras de Farmacia y Bioquímica de la Universidad de Buenos Aires. En trabajos previos se individualizaron varios obstáculos que dificultaban los procesos de enseñanza y aprendizaje de Química Orgánica y se implementaron algunas estrategias para intentar superarlos (Blanco et al., 1998 a y b). En función de los resultados obtenidos, se focalizó la atención en el tipo de actividades y en los materiales impresos (guías de estudio y cuestionarios) utilizados como recursos didácticos en el trabajo en el laboratorio y en las clases de problemas. Se encontró que los mismos ya no resultaban adecuados pues apuntaban a un aprendizaje de tipo memorístico, resultando poco atractivos para los alumnos. Esto, a juicio de los autores, llevaba a un menor nivel de compromiso de los estudiantes en el proceso de aprendizaje.

El presente trabajo está dedicado al replanteamiento de las actividades en clases de resolución de problemas y de trabajos prácticos de Laboratorio y a la reformulación de los materiales impresos editados por la Cátedra, de acuerdo con las nuevas actividades. Para esto, se toma como ejemplo el tema "Síntesis Orgánica". Se realiza una selección y jerarquización de los contenidos a enseñar, definiendo los macroconceptos estructurantes de la unidad. En la elaboración del nuevo material impreso se intenta superar las deficiencias de los cuestionarios empleados hasta ese momento. Se plantean problemas que permiten trabajar sobre los macroconceptos básicos, relacionados con los posibles contextos en que se desempeñarán los profesionales en el ámbito de la industria farmacéutica, la farmacia oficinal, la investigación y/o en la docencia. Se intenta además plantear actividades abiertas, coherentes con la creatividad del trabajo científico (Furió et al., 1994) y que despierten el interés de los estudiantes (Gil, 1991).

\section{METODOLOGÍA}

Se pusieron en práctica las siguientes estrategias: a) Reformulación de los ejercicios de la Guía de Problemas como verdaderos problemas (Perales, 1993), representativos de los que pueden plantearse en la práctica profesional; y b) Replanteamiento del trabajo en el laboratorio, teniendo en cuenta que el quehacer profesional implica permanentemente la toma de decisiones.

\section{a) Reformulación de la Guía de Problemas}

La unidad se estructura en torno a cuatro macroconceptos interrelacionados: 1) Escala y equipamiento, 2) El profesional como solucionador de problemas, 3) Síntesis estéreo-selectiva, 4) Propiedades de los compuestos orgánicos. Siguiendo este esquema, se reformulan los ejercicios como verdaderos problemas:

- Escala y equipamiento

1.- Usted necesita sintetizar $2 \mathrm{~g}$ de aspirina y dispone de las siguientes materias primas: benceno, tolueno, o-cresol, ácido acético, cloruro de acetilo, ácido sulfúrico, hidróxido de sodio, permanganato de potasio, piridina y anilina.

a) ¿Qué rutas sintéticas son posibles? Establezca ventajas y desventajas de cada una. ¿Cuál elegiría y por qué?

b) Esquematice el/los aparato/s que necesitaría para la síntesis propuesta por Ud. en a).

c) Compare sus esquemas con los que diseñó su compañero y discútalos.

2.- Si en cambio Ud. trabaja en la industria y le encargan sintetizar $100 \mathrm{~kg}$ de aspirina:

a) ¿Emplearía la misma secuencia sintética que la propuesta en la pregunta 1) ¿Por qué? Sugerencia: consulte la literatura correspondiente a métodos industriales.

b) ¿Utilizaría el mismo equipo? En caso negativo, indique qué otro emplearía. Sugerencia: consulte la literatura correspondiente a equipamiento industrial.

3.-¿Ingeriría la aspirina que acaba de sintetizar? ¿Por qué? ¿Qué controles indicaría Ud. hacerle?.

- El profesional como solucionador de problemas 
1.- Frente a un alerta farmacológico en el mercado, se suspende momentáneamente el consumo de aspirina, reemplazándola por ibuprofeno o paracetamol.

a) Plantee una posible ruta sintética para cada uno de los analgésicos mencionados.

b) Contraste las rutas diseñadas por Ud. con las que halló en la literatura.

2.- Trabajando en una industria alimentaria dedicada a la fabricación de conservas, surge el inconveniente de que el conservante que se utilizaba está vencido. Es preciso reemplazarlo inmediatamente por butilhidroxitolueno (BHT) o butilhidroxianisol (BHA).

a) Diseñe la síntesis de uno de ellos, teniendo en cuenta que dispone de benceno (como producto de partida), y de los reactivos que necesite.

b) ¿En qué concentración aconsejaría agregarlo a la conserva? Tenga en cuenta: la pureza, los límites de toxicidad y las recomendaciones de la FAO.

\section{- Síntesis asimétrica}

1.- En el área de fabricación de dulces dietéticos de la misma industria alimentaria, utilizaron para endulzar una mermelada el contenido de un tambor rotulado como $( \pm$ ) ASPARTAMO (Nutrasweet $(\AA)$. La mermelada resultó con un fuerte sabor amargo, arruinándose la partida. ¿A qué pudo deberse este problema?

2.- ¿Cómo procedería para hacer utilizable el contenido del tambor para endulzar?

3.- ¿Podría obtener el (-) aspartamo ópticamente puro de otra manera?

4.- Mencione dos ejemplos donde, en la industria farmacéutica, puedan cometerse otros errores del tipo del cometido en 1) pero con resultados más severos.

- Propiedades de los compuestos orgánicos

1.- ¿A qué se deben las propiedades antioxidantes de conservantes como BHT y BHA? ¿Cuál es el mecanismo asociado a esta propiedad?

2.- Justifique en qué condiciones almacenaría BHT y aspirina, marcando la/s opción/es que considere correctas:

En lugar oscuro y húmedo

En lugar luminoso y húmedo

En frasco color ámbar hermético

En desecador a la luz

En desecador a oscuras

En desecador con ácido sulfúrico

3.- Indique qué método emplearía y en qué condiciones lo haría, para purificar la aspirina sintetizada por Ud., teniendo en cuenta las impurezas que considera que posee.

4.- Habiéndose perdido el rótulo del envase donde guardó la aspirina, describa cómo podría comprobar si aún es apta para el consumo (como sugerencia, tenga en cuenta la estabilidad del medicamento, es decir, los posibles mecanismos y productos de degradación).

5.- ¿Cuáles serían los inconvenientes asociados al uso excesivo de edulcorantes tanto de la familia de los ciclamatos como de aspartamo? Sugerencia: consulte la literatura referida a toxicidad, metabolismo y productos de degradación de los edulcorantes mencionados.

b) Replanteamiento de actividades para el trabajo en el Laboratorio 
Se elige para este fin dos de las cuatro clases de Trabajos Prácticos correspondientes al tema "Síntesis Orgánica". En cada clase los grupos tendrán trabajos diferentes pero claramente definidos, como se describe a continuación.

\section{Primera Clase}

Parte I: Síntesis de laboratorio de ácido acetilsalicílico (Aspirina ${ }^{\circledR}$ ) en distintas condiciones experimentales. Los alumnos se dividirán en cinco grupos, cada uno de los cuales ensayará la síntesis de aspirina según las siguientes indicaciones:

Grupo 1: siguiendo la técnica descripta en la Guía.

Grupo 2: omitiendo el catalizador (ácido sulfúrico).

Grupo 3: reduciendo el tiempo de reacción a la mitad.

Grupo 4: omitiendo la recristalización.

Grupo 5: duplicando el tiempo de reacción.

Para analizar la influencia de estos factores, se compararán entre grupos los siguientes parámetros: a) Rendimiento de la reacción, b) Punto de fusión del producto obtenido. La discusión y puesta en común de los resultados será coordinada por el docente.

Parte II: Estabilidad química de la aspirina. Cada uno de los grupos almacenará, hasta la semana siguiente, una porción de la aspirina sintetizada por el Grupo 1 (sintetizada y purificada según la técnica de la guía), en distintas condiciones:

Grupo 1: en frasco abierto.

Grupo 2: en frasco color caramelo cerrado, en lugar fresco.

Grupo 3: en frasco abierto dentro de la heladera.

Grupo 4: en un desecador con ácido sulfúrico.

Grupo 5: en un desecador con agente deshidratante a elección.

\section{Segunda Clase}

Cada uno de los grupos realizará lo siguiente: 1) Identificará los agentes físicos y/o químicos a los que estuvo expuesta la aspirina en cada una de las condiciones empleadas por los distintos grupos para almacenar la muestra (luz, calor, agua, oxígeno atmosférico); y 2) Analizará la estabilidad del ácido acetilsalicílico en cada una de las condiciones en las que los distintos grupos almacenaron la muestra. Para esto, los grupos tomarán una porción de cada una de las muestras, y emplearán las siguientes metodologías de análisis:

Grupo 1: métodos cromatográficos (tlc).

Grupo 2: métodos físicos (punto de fusión).

Grupo 3: métodos espectroscópicos (IR, UV).

Grupo 4: espectroscopía RMN de ${ }^{1} \mathrm{H}$ y ${ }^{13} \mathrm{C}$.

Grupo 5: métodos químicos (ensayos de grupos funcionales).

Para efectuar el análisis, dispondrán de testigos tanto de aspirina como de los posibles impurificantes y productos de degradación de la misma. Cada grupo solicitará al docente el/los testigos que considere necesarios. La puesta en común y discusión de los resultados obtenidos será coordinada por el docente. Los resultados serán contrastados con la literatura. Al cabo de esta segunda clase cada alumno entregará un informe con los resultados obtenidos por cada grupo, su interpretación, discusión y conclusiones. 


\section{RESULTADOS Y DISCUSIÓN}

Para evaluar los resultados de la implementación de los nuevos recursos, se realizaron las observaciones correspondientes a la Clase de Resolución de Problemas y a las clases de trabajo en el Laboratorio de acuerdo a la nueva planificación.

\section{Clase de Resolución de Problemas}

Cada docente dividió a su Comisión en dos grupos. Un grupo trabajó con el cuestionario viejo y el otro grupo con el nuevo. Se observó que la nueva formulación de los problemas suscitó el interés de los alumnos, que se involucraron activamente en su resolución. Dado que este tipo de tareas implica mayor cantidad de tiempo para su realización, el grupo que trabajó con la guía tradicional finalizó antes la actividad y, contrariamente a lo que suele suceder en esas circunstancias, se unieron al primer grupo y comenzaron a participar de la discusión. Una vez finalizada la clase varios alumnos se acercaron al docente pidiendo más ejercitación de este tipo.

\section{Trabajo en el Laboratorio}

La mayoría de los alumnos, una vez explicadas las consignas, prefirió incorporarse al Grupo 1. En los grupos 2 y 5 se observaron mayores resistencias, porque los alumnos del Grupo 2 sabían de antemano que sin catalizador probablemente no obtendrían un rendimiento adecuado del producto, y los del Grupo 5 tardarían el doble de tiempo. No obstante esto, cuando se realizó la puesta en común todos los alumnos participaron activamente en la discusión y confección del cuadro final.

Uno de los mayores desafíos que presenta la enseñanza universitaria de materias básicas es lograr que los alumnos visualicen las posibles aplicaciones de los contenidos a aprender. Es sabido que el aprendizaje se hace menos atractivo cuando no se conoce la utilidad de lo que se va a estudiar (Gil, 1991). El tipo de actividades que se plantean, que sitúa a los alumnos dentro del contexto de su futuro desempeño como profesionales, contribuyó a que los mismos se involucraran más activamente en el proceso de aprendizaje. Por otra parte, a menudo los estudiantes conocen la información relevante que les permitiría resolver una situación problemática, pero no son capaces de aplicarla en forma espontánea (Monereo, 1994). Se observó que la resolución de este tipo de problemas estimuló la puesta en acción de los conocimientos conceptuales previamente adquiridos, y las actividades planteadas para el laboratorio favorecieron la aplicación de dichos conocimientos y de las habilidades desarrolladas en la resolución de problemas hipotéticos al contexto del trabajo en el laboratorio.

Se habían notado previamente dificultades en los alumnos para distinguir entre métodos sintéticos "de laboratorio" e "industriales", así como también las diferencias en el equipamiento necesario para llevar a cabo la síntesis en cada caso. En este sentido, el tipo de ejercitación propuesta pone de manifiesto esa diferencia y hace que el alumno deba recurrir necesariamente a la literatura. De esta manera se estimula la consulta y el análisis de libros de texto.

\section{CONCLUSIONES}

A partir del análisis y la discusión de los resultados obtenidos, se plantean las siguientes conclusiones:

a) El replanteamiento de los ejercicios como verdaderos problemas y su contextualización dentro del ejercicio profesional, contribuyeron a aumentar la motivación de los alumnos, factor imprescindible para encarar una situación de enseñanza-aprendizaje.

b) Las actividades propuestas estimularon la comprobación y la puesta en acción de los conocimientos conceptuales desarrollados en clases teóricas, así como su aplicación estratégica a situaciones problemáticas concretas.

c) Las nuevas actividades enfatizaron las diferencias entre métodos de laboratorio e industriales. 
d) A juicio de los autores de este trabajo, la destreza adquirida en la resolución de problemas de química orgánica trasciende las situaciones de aplicación de los enunciados de los mismos, para ser potencialmente aplicable a otros problemas reales o hipotéticos de la vida estudiantil o profesional.

\section{AGRADECIMIENTOS}

Los autores (as) agradecen a la Bioq. María Cristina Caterina, quien permitió la observación de sus clases.

\section{REFERENCIAS}

Blanco, M. y otros ocho autores; "Formación de nuevos docentes para atender cursos universitarios masivos de química orgánica". Información Tecnológica: 9 (6), 199 (1998a).

Blanco, M. y otros cuatro autores; "Obstáculos detectados en la enseñanza universitaria. Algunas estrategias y propuestas". Información Tecnológica: 9 (6), 193 (1998b).

Furió, C.J. y otros dos autores; "Contribución de la resolución de problemas como investigación al paradigma constructivista del aprendizaje de las ciencias". Investigación en la Escuela: 24 (1994).

Gil, D.; “¿Qué han de saber y saber hacer los profesores de ciencias?”. Enseñanza de las Ciencias: 9 (1), 69 (1991).

Monereo, C.; "Estrategias de enseñanza y aprendizaje. Formación del profesorado y aplicación en la escuela". Ediciones Grao, Barcelona (1994).

Perales, F.J.; "La resolución de problemas: una revisión estructurada". Enseñanza de las Ciencias: 11 (2), 170 (1993). 